\title{
Agents and Coordination Artifacts for Feature Engineering
}

\author{
Alessandro Ricci \\ DEIS \\ Università degli Studi di Bologna a Cesena \\ Via Venezia, 52 - 47023 Cesena (FC) Italy \\ mailto:aricci@deis. unibo.it
}

\begin{abstract}
Agents and Multi-Agent Systems (MAS) are considered a suitable paradigm for engineering complex systems. Interaction is a primary source of this complexity and coordination plays a key role for its management, providing the means for modelling and shaping the agent interaction space so as to engineer the global behaviour of the system as a whole. Feature-based systems are certainly complex systems: they are generally composed by several interrelated parts which work together to provide global functionalities. The set of functionalities is not fixed, but typically evolves with the (dynamic) integration of new features. In this paper we investigate the possibility of using agents and coordination abstractions for the engineering of feature-based systems; in particular, typical feature issues - such as feature plug-and-play and the feature-interaction problem - are framed in the agent-based engineering context.

The content of the paper is articulated as follows: First, we provide an overview of agent oriented software engineering, in particular of the reason why agent paradigm is suitable for complex system. Here, the importance of the coordination dimension is reminded, in particular the adoption of suitable coordination artifacts to engineer collective behaviour of MAS is discussed. Then, features and feature-based systems are briefly considered, sketching some of the main characteristics which make them complex systems. After that, a perspective for engineering feature-based systems on top of agents and coordination artifacts is provided; the main points of the discussion are exemplified using the TuCSoN MAS coordination model \& infrastructure applied to a simple case study typically found in the feature literature, an email service engineering.
\end{abstract}

\section{Agents and Coordination for Complex System Engineering}

Agent and Multi-Agent System (MAS) paradigms are considered the right level of abstraction for modelling and engineering complex systems, characterised by organisation structures and coordination processes that are more and more articulated and dynamic [22, 23]. Agent-based control systems, inter-organisational workflow management systems, agent-based Computer Supported Cooperative 
Work (CSCW) and team-based cooperative military contexts are prominent examples.

For complex systems we mean here systems composed by a quite large number of independent and interacting parts, typically immersed and distributed into an unpredictable/open environment (physical or non physical, such as the Internet). The openness of systems typically implies that both the set of the components of the systems, and their relationships/interaction can change dynamically, during the lifetime of the systems themselves. Distribution and concurrency are characteristics that can be found in almost any complex system.

The agent and multiagent paradigms are considered suitable since they provide abstractions (and methodologies, technologies) which naturally account for these issues. Various definitions has been given for the agent concept. An agent can be defined as an encapsulated computer system that is situated in some environment and can act flexibly and autonomously in that environment to meet its design objectives [23]. Among the main agent characteristics largely described in literature here we mention [7]:

- autonomy - agents have control over both their internal state and their own behaviour. This implies that an agent embeds necessarily its own control flows.

- situatedness - agents are immersed in a particular environment, over which they have a partial control and observability: they can receive inputs related to the state of their environment through sensors and they can act on the environment through effectors;

- goal orientation - agents are designed to fulfill tasks related to some specific role, in order to achieve some specific objective.

Other important characteristics are pro-activeness, that is the ability to opportunistically adopt goals and take the initiative, and reactivity, that is the ability to respond in a timely fashion to changes that occur to their environment.

It is worth remarking here some of the reasons why the agent paradigm is considered more adequate for engineering complex systems than other mainstream paradigms, such as the object-oriented and component-oriented ones [8]. Issues such as distribution of control, concurrency, openness of the environment - which characterise complex systems - are directly addressed by the abstractions provided by the agent paradigm; this is generally not true in the case of object-oriented and component-oriented models. Of course, the same issues can be faced also in the context of these paradigms, but typically by adopting nonorthogonal mechanisms which cannot found a suitable specification in the computational model of the paradigm. Conversely, object-oriented and componentoriented technologies are typically used to implement agent-based technologies.

Multi-Agent Systems are ensemble of agents, acting and working independently from each other, each representing an independent locus of control of the whole system. Each agent tries to accomplish its on task(s) and, in doing so, will typically need to interact with other agents and its surrounding environment, in order to obtain information / services that it does not posses or to coordinate its activities in order to ensure that its goals can be met. However, a 
multi-agent system, as a whole, is conceived to achieve more complex or wider goals than the mere sum of its component agents goals: MASs can typically be understood as societies of agents, where the mutual interactions between agents and with their environment leads to a useful global behaviour [2]. For this reason, another fundamental characteristic of the agent abstraction is the social ability, as the capability to be part of an agent society. The two main issues concerning MASs are organisation and coordination, two interrelated dimensions that are fundamental for designing, building and enacting Multi-Agent systems. Roughly speaking, organisation accounts for defining MAS structure and rules concerning this structure, often in terms of roles, and agent-role, inter-role and role-environment relationships; coordination accounts for defining the social rules and norms necessary to manage agent interaction and drive the MAS to achieve its social objectives.

In this context, MAS infrastructures are fundamental to support agent societies lifecycle, providing basic services to support both intra-agent and interagents issues, such as agent life-cycle management, agent communication and interoperability, and high-level services for MAS organisation and coordination $[4]$.

\subsection{Engineering Systems with Agents}

So, agent and MAS paradigm provides models, infrastructure and technologies that can be exploited to cover all the stages of software engineering, from analysis to deployment and runtime. Basically, engineering a system in terms of agents involves first identifying individual and collective/social activities needed to achieve system goals, and then mapping the activities on agents and agent societies according to some organisation and coordination approach [10,24]. In particular, individual tasks are associated with one specific competence in the system, related to the need to access and effect a specific portion of the environment, and carry out some specific job. Each agent in the system is assigned to one or more individual tasks, and the agent assumes full responsibility for carrying out assigned tasks. From an organisational perspective, this corresponds to assigning each agent a specific role in the organisation/society. Social tasks represent the global responsibilities of the agent system, typically requiring several heterogeneous competencies. The achievement of social tasks leads to the identification of global social laws that have to be respected and/or enforced by the society of agents, to enable the society itself to function properly and according to the global expected behaviour. From an coordination perspective, this corresponds to choose a coordination model and use the abstractions it provides to represent and enact the social laws in terms of coordination laws shaping and ruling the agent interaction space [10].

\subsection{Coordination as Engineering Dimension}

Coordination in then a fundamental engineering dimension of MAS. Coordination approaches can be categorised in subjective and objective [13]: in the former, 
all the coordination burden related to the achievement social tasks is distributed upon agents (no mediation abstractions are used); in the latter, the coordination burden is balanced between agents and special purpose coordination artifacts, which are runtime coordination abstractions mediating agent (inter)action, and specifically designed to help an agent society to achieve its objectives. The behaviour of a coordination artifact is designed to embed and enact the social laws as required to achieve a social task.

The notion of coordination artifacts supporting societies in their coordination activities can be found in different disciplines, also outside computer science. According to Activity Theory, every collaborating activity occurring in any social context is always mediated by some kind of tool (artifact), in order to manage the complexity of the involved interaction and automate the coordination [20]. Coordination artifacts are meant to be provided and supported by the MAS infrastructure, according to the coordination as a service perspective [21]: so the infrastructure itself must provide agents not only enabling services - enabling for instance communication and inter-operability -, but also governing ones, to specify and enact norms and rules governing agent interaction. The infrastructure then can be provider also of the basic services required to use and manage coordination artifacts - in terms of creation, inspection and adaptation of their behaviour.

Summing up, engineering complex systems upon a MAS infrastructure supporting an objective coordination approach basically accounts for assigning and enacting individual tasks by means of individual agents, and using suitably engineered coordination artifacts to support social tasks and collaborating activities.

\subsection{TuCSoN Coordination Infrastructure}

TuCSoN is an example of MAS coordination infrastructure supporting objective coordination [16]. An overview of the TuCSoN world is depicted in Figure 1. In TuCSoN coordination artifacts take the form of tuple centres, design / runtime coordination abstractions provided to agents by the infrastructure in order to enable and govern their interaction [12]. More precisely, tuple centres are programmable tuple spaces [12], a sort of reactive logic based blackboards; agents interact by writing, reading, and consuming logic tuples - ordered collections of heterogeneous information chunks - to/from tuple centres via simple communication operations ( out, rd, in) which access tuples associatively (see Figure 2 ). While the behaviour a tuple space in response to communication events is fixed and pre-defined by the model, the behaviour of a tuple centre can be tailored to the application needs by defining a suitable set of specification tuples, which define how a tuple centre should react to incoming/outgoing communication events. The specification tuples are expressed in the ReSpecT language [3]; ReSpecT is logic-based, Turing-equivalent, and makes it possible to express coordination laws in the form of reactions: it can be considered an assembly language for managing interaction and doing coordination. The reactions are triggered by interaction events concerning the tuple centres (both external, such 

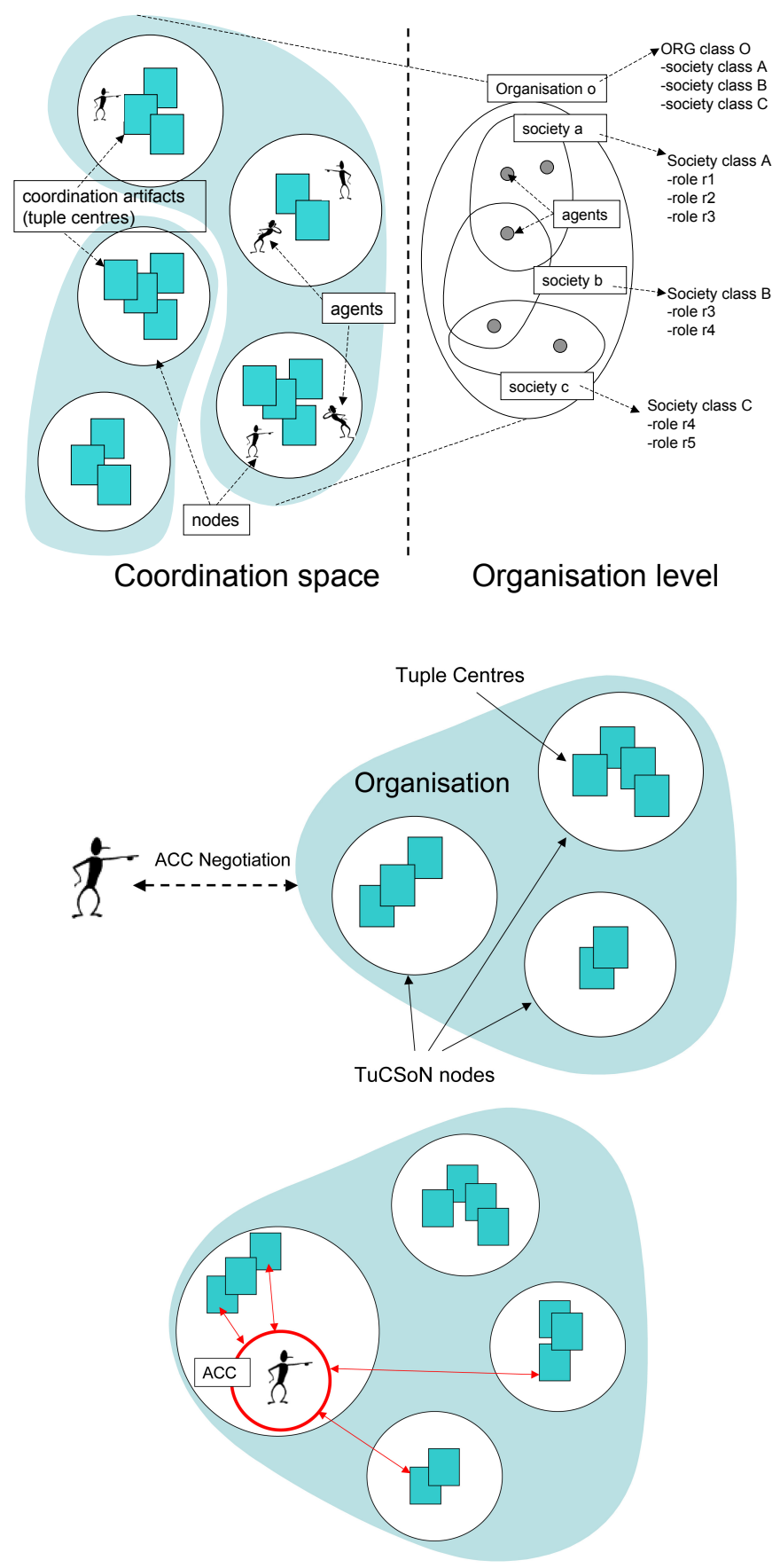

Fig. 1. TuCSoN world. (Top) TuCSoN coordination space, with coordination nodes and tuple centres inside the nodes. (Middle) Negotiation of an Agent Coordination Context (ACC) by an agent that aims at joining an organisation and using its tuple centres. (Bottom) Once inside the organisation, the agent use the ACC as interface to access tuple centres. 

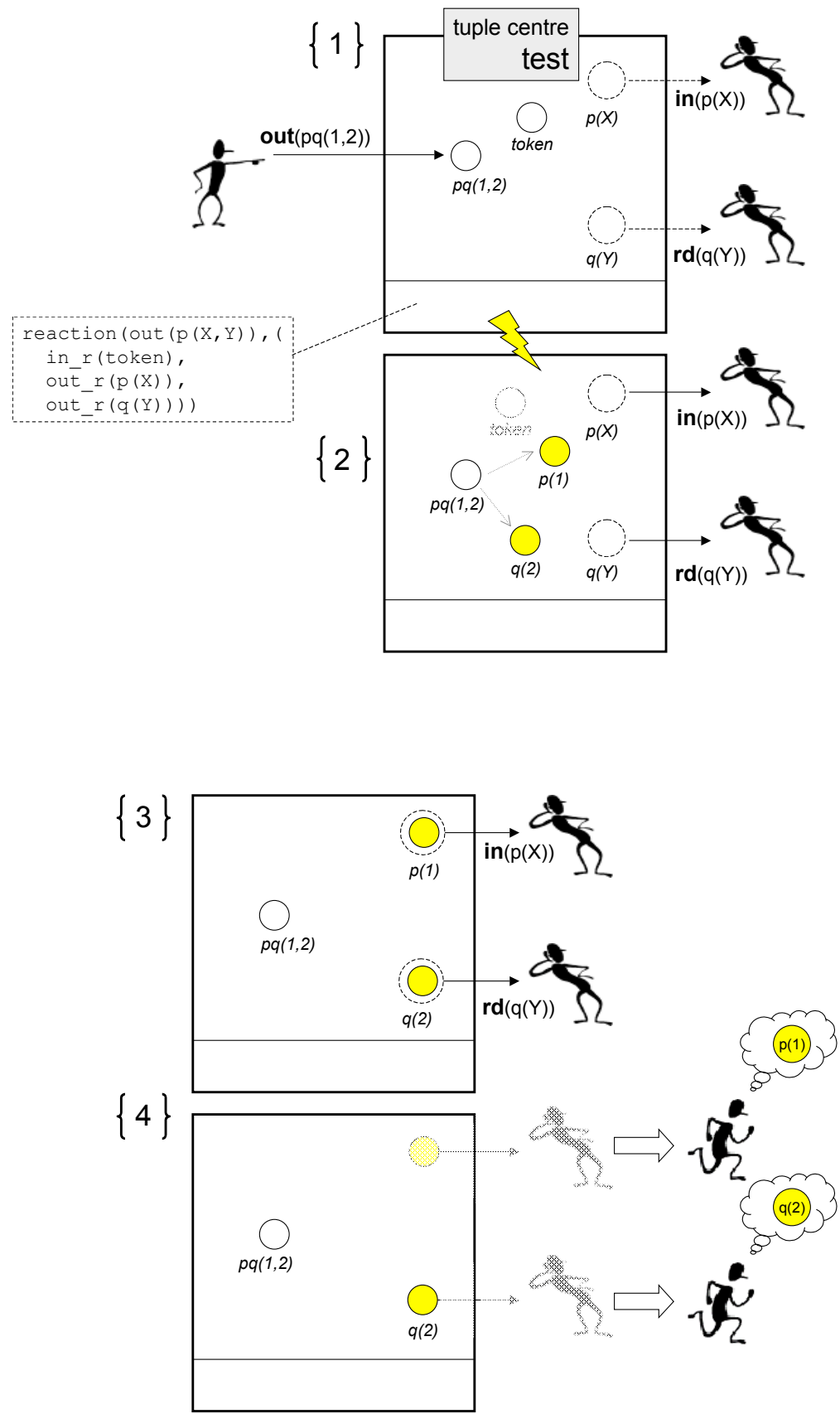

Fig. 2. Using Coordination Artifacts. In the example, three agents interact through the Test tuple centre. Four consequent stages are shown: (1) an agent inserts a tuple $\mathrm{pq}(1,2) ;(2)$ the tuple centre reacts according to the reaction shown in the picture, removing the tuple token and inserting the tuples $p(1)$ and $q(2) ;(3)$ and (4) two agents that were waiting for tuples matching the $\mathrm{p}(X)$ and $\mathrm{q}(Y)$ templates respectively retrieve and read the tuples just inserted. 
as the invocation of a coordination primitive by agents, and internal, such as the insertion or removal of a tuple by means of a ReSpecT primitive). The semantics of ReSpecT has been formally specified [11], defining the behaviour of the language primitives and the properties of the reaction triggering and execution stages (such as non-determinism and the atomic/transactional behaviour).

So, tuple centres can be seen as general-purpose customisable coordination artifacts, whose behaviour can be dynamically specified, forged and adapted.

From the topology point of view, tuple centres are collected in TuCSoN nodes, spread over the network and belonging to specific organisations (see Figure 1, top). In order to access and use tuple centres of an organisation context, an agent must negotiate and enter an Agent Coordination Context, which is used to define its presence / position inside the organisation in terms of allowed actions on the available artifacts (tuple centres) [15]. Figure 1 shows these two basic stages: first an agent negotiates the configuration of the ACC by means of the services provided by the infrastructure (Figure 1, middle); then, if the agent request is satisfiable (according to the organisation rules defined by the specific organisation context), an ACC with the specific configuration is created and entered logically by the agent [14] (Figure 1, bottom).

Summing up, in this section we provided an overview of the agent and coordination artifact abstractions for engineering complex systems. In next section we will consider feature-based systems as complex systems, and then amenable to be engineered using the agent oriented approach.

\section{Feature-Based Systems as Complex Systems}

Features can be defined as optional-bits of self-contained functionalities, and are used as structuring mechanism for engineering systems. Originally, the concept has emerged in telecommunication context, in particular in telephone systems analysis as a way of describing optional services to which telephone users may subscribe [9].

Engineering a system by means of the feature abstraction involves starting with a basic system core and then completing/extending the systems by adding new features, as self-contained functionalities. Typically features cannot be represented directly neither as objects, nor as components, since they concern functionalities which span on multiple objects/modules/components. We can recognise in the feature approach also a methodological aim: a feature includes a description of how to transform a base system, by adding new encapsulated functionality; the challenge is then the capability to add this functionality which typically affects many parts of the system without rewriting/changing all the system parts.

Feature integration and composition are then one of the main issues, and can be described as the process of adding a feature to a base system. This process is clearly non-monotonic, since a new feature may override behaviour / properties present in the base system and in other features. 
From the integration issues, it emerges the main problem challenging research on feature-based systems, that is the feature interaction problem [1], when several features are integrated in the same system, they may interfere with each other in undesirable ways. Conversely, in the same integration context, features can work together positively: that is there is feature inter-working, obtaining new functionalities beyond the sum of the individual features. So detecting feature interaction that creates interference and not inter-working is a problem of primary importance. It is worth noting that in other computer science contexts (such as Software Engineering, Artificial Intelligence, Programming Languages) the term interaction is used with a different acceptation, not as interference but as the (usually desirable) effect of dependencies among components of a system; in particular, it is used often to indicate communication (even if communication can be considered as a special form of interaction). So, we can devise two different perspectives on the notion of interaction: from the one side, each feature emerges from the (positive) interactions of the components of a system: in fact, the functionalities encapsulated by a feature can be often achieved only by suitably coordinating the communications and more generally the dependencies among the components. On the other side, the interaction between features is instead what must be avoided or controlled.

Other interesting issues concerning feature research and fundamental for the engineering of systems are:

- feature models and languages - defining models and languages for specifying and describing features. What computational models are useful for capturing feature abstraction? Either using purely algorithmic languages or object-oriented ones seem not to be adequate to capture the real essence of features, as functionalities involving multiple entities. For this reason Aspect Oriented paradigm seems to be more effective; however, this approach seems not to be adequate for handling feature interaction and inter-working issues; moreover, Aspect Oriented focuses mainly at the compile time and hardly provide means to support runtime/dynamic integration/evolution of features. Policy-driven models have been recently considered as an other possible paradigm, mapping feature as policies that rule interaction components of a system and provide in the overall the functionalities required. Here we claim that also coordination models and languages $[5,17]$ can be considered interesting for modelling and expressing features. These approaches promote a clear separation between computation from coordination issues, and provide specific abstraction for specifying and enacting the latter; the coordination dimension concerns interaction management, and is not related to a specific component but to the laws that glue components together;

- formal feature - the possibility of defining a formal semantics of feature model and languages is fundamental for the verification of formal properties, in particular to face the feature interaction problem with formal tools;

- feature evolution - engineering systems with some degree of openness implies the need of supporting not only the integration of new features, but also 
the evolution/change/replacement of existing ones, without breaking system consistency;

- feature for concurrent systems - almost all feature-based application scenarios include aspects concerning concurrency and distribution, where features concern components distributed on different execution contexts, on different nodes of a network, so with multiple control flows;

- feature infrastructure - modern application scenarios require also more and more the capability of changing systems behaviour at runtime, dynamically; this implies the capability of integrating, removing, evolving features at runtime, toward runtime feature plug-and-play $[18,19]$. This capability typically can be faced by means of proper software infrastructures, providing runtime support (services) for manipulating abstractions (features in this case) as first class entities also at runtime.

\section{Engineering Features with Agents and Coordination Artifacts}

Since agents and coordination abstractions are good for complex systems engineering and feature-based systems provide a high degree of complexity, it is interesting to investigate the application of the former as engineering tool for the latter. Accordingly, this section provides a perspective, a possible way to conceive the engineering of feature within a MAS context, in particular discussing the main points and challenges that concern feature based systems as sketched in the previous section.

As mentioned in Section 1, agent oriented software engineering accounts for identifying in the analysis stage individual and social tasks required to reach system objectives. Individual tasks are mapped onto individual agents and the social tasks with norms and global properties of the system are mapped onto agent societies or groups, which adopt some kind of coordination artifacts in order to achieve the social objectives. A feature encapsulates a functionality concerning multiple components inside a system; then it comes natural to recognise the feature concept as a social task, which can be achieved by suitably adapting existing coordination artifacts, and possibly introducing new agents providing specific competencies that the features require. In other words, a new feature implicitly or explicitly leads to the identification of new relationships and rules among a set of existing components or activities, and possibly to the definition of new specific activities (individual tasks): so, first the new relationships and rules can be expressed by coordination laws extending the behaviour of the coordination artifact gluing existing individual activities, and then the new individual tasks are assigned to new agents (roles).

Given this conceptual mapping, feature plug-and-play is obtained by evolving dynamically the behaviour of the coordination artifacts, and by possibly introducing new agents, responsible of the execution of some new individual task related to the new feature. If the system is well-engineered, the integration 
of a new feature would not imply changing existing agents, but only the glue aggregating and coordination their activities.

So, the feature interaction problem can be faced focusing primarily on the coordination artifacts behaviour, checking for the presence of coordination laws which interfere each other in an undesirable way. In this framework, feature interaction emerges from dependencies that have been not properly handled in the coordination medium. The same reasoning applies for the opposite issue, that is feature inter-working: possible desirable interaction among features can be here obtained and managed by suitable coordination laws which do not interfere, but work together fruitfully. For all the other issues:

- feature models and languages - in this case the model and language used to define and enact features is strictly related to the coordination model (and language) adopted to support multi-agent system coordination, in particular to define coordination artifacts and their behaviour. For instance, in the case of TuCSoN a feature would be mainly described in terms of coordination laws expressed in the ReSpecT language;

- formal feature - coordination models and languages with formal semantics can be suitable for investigating the verification of formal properties of coordination. This is the case of tuple centre model and ReSpecT language, for instance. In this context, this accounts for verifying formal properties of features, mapped on top of coordination laws enacted by media. This possibility is a key to face the feature-interaction problem from a formal point of view;

- feature evolution - feature evolution is supported in this case by the capability of changing dynamically the behaviour of the coordination artifacts by changing/adapting the coordination laws concerning the feature. In the case of TuCSoN, for instance, the behaviour of a tuple center can be inspected and changed dynamically by means suitable tools (for humans) or by coordination primitives that can be used directly by agents;

- feature infrastructure - any model based on agents and coordination artifacts necessarily requires the notion of infrastructure to support at runtime these abstractions and their interactions, by means of suitable services. Then, the infrastructure has a key role in supporting issues related to integration and evolution of features, in particular by providing the services to inspect and change at runtime the behaviour of the coordination artifacts used for implementing them;

\section{A Simple Example: Engineering Email Service Features with TuCSoN}

In this section we exemplify the approach described in previous section using TuCSoN to engineer a simple case study typically found in feature literature $[6$, 


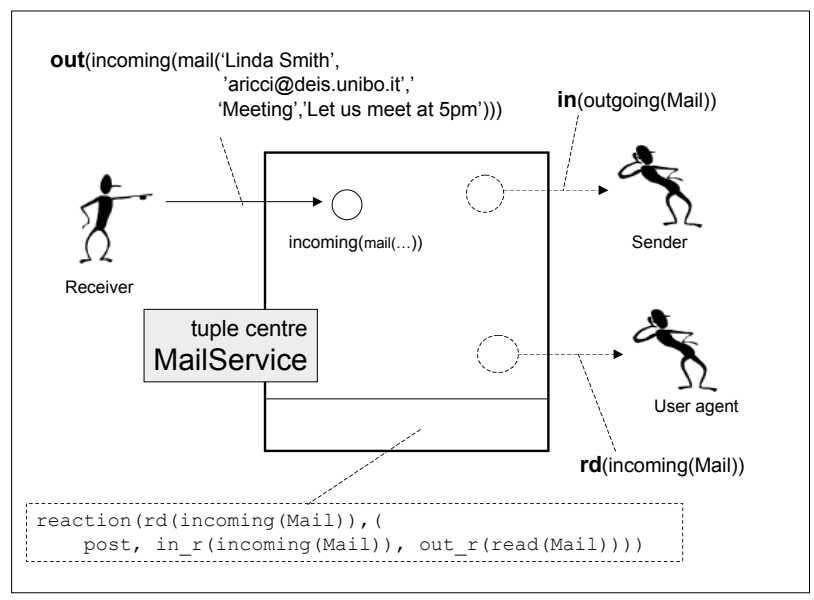

Fig. 3. The basic email service engineered with TuCSoN. Three agents are responsible for individual task (sending emails, receiving emails and assisting the human user) and the MailService tuple centre is used to support their coordination.

19], an email service ${ }^{1}$. The objective of our system is to setup an email service, which can be used both from human and artificial agents. The functionalities of the basic system concern receiving, sending and visualising (for humans) emails. The core service will be then extended by integrating new features, the encryption/decryption capability and the auto-reply functionality. With a simple analysis, three basic roles can be identified:

- the receiver role, responsible of receiving incoming emails though of the POP protocol/infrastructure;

- the sender role, responsible of sending outgoing emails using of the SMTP protocol/infrastructure;

- the user interface role, responsible of assisting users in checking and visualising incoming emails, and composing outgoing emails.

The social laws glue the activities of these roles fruitfully. The roles are assigned and played by TuCSoN agents, with the capabilities required to fulfill role tasks. In order to coordinate the individual activities, we use a single tuple centre, called MailService. An overall picture of the basic system is depicted in Figure 3. We use the logic tuple

\footnotetext{
${ }^{1}$ the source code presented in this section can be tested by using TuCSoN technology, available as open source project at http://lia.deis.unibo.it/research/tucson or http://tucson. sourceforge.net
} 
reaction(rd(incoming (Mail)), (

post, in_r(incoming (Mail)), out_r(read(Mail)))).

Table 1. ReSpecT code defining the basic coordination behaviour of the MailService tuple centre. Basically, in order to avoid that the same incoming mail could be read more than once, when an incoming tuple is read by means of the rd operation, the tuple is removed and a new tuple read with the same mail information is inserted.

mail (Sender, Receiver, Subject, Content)

to represent email information, where simple string can be used to describe all the fields of the mail. An example of mail tuple can be:

mail ('Linda Smith', 'aricci@deis.unibo.it', 'Meeting',

'Let us meet at $5.00 \mathrm{pm}$. Bye, Linda')

The presence of incoming and outgoing emails are represented respectively by tuples incoming(Mail) and outgoing(Mail), where Mail follows the format described above. Receiver agents notify the arrival of a new email to the agent society by placing a new incoming tuple in the MailService tuple centre (by means of an out operation). Sender agents collect outgoing tuples by means of in or inp operation as they appear in the tuple centre, and use the information to send emails via the SMTP protocol. User agents collect and visualise new incoming emails for a human user by reading incoming tuples through the rd operation, and also assist a human user in sending emails by inserting outgoing tuples with proper data. The synchronisation capabilities provided by the basic coordination primitives (in, out, etc.) are sufficient for realising the simple coordination required by the basic service core. In addition, we add a coordination law to avoid that incoming emails could be read and visualised more than once, by turning incoming (Mail) tuples in read (Mail) tuples as soon as the incoming tuples are read the first time. In order to enact this coordination behaviour, the tuple centre MailService is programmed with the reaction shown in Table 1.

\subsection{Handling Feature Integration: Encryption and Decryption}

Now we aim at extending the basic system core by integrating the encryption/decryption feature [6]. Abstracting from details, we suppose that format of the logic tuple deployed for an encrypted email is:

mail (Sender, Receiver, encrypted (Subject), encrypted(Content)) 
reaction (out (outgoing (Mail)), (

rd_r(encryption(true)), in_r(outgoing(Mail)),

out_r(mail_to_encrypt (Mail)))).

2

reaction (out (mail_encrypted(Mail)), (

in_r(mail_encrypted(Mail)), out_r(outgoing(Mail)))).

reaction (out (incoming (mail (,$R$, encrypted (Subj), encrypted (C))) ), (

in_r (incoming (mail (S, R, encrypted (Subj), encrypted (C)))),

out_r(mail_to_decrypt (mail (S,R, encrypted (Subj), encrypted(C)))))).

4

reaction(out (mail_decrypted(Mail)), (

in_r(mail_decrypted(Mail)), out_r(incoming(Mail)))).

Table 2. ReSpecT code defining the encrypt (top) and decrypt (bottom) feature.

In order to add the encryption feature we extend the basic societies by adding an agent (role) able to encrypt information, and the coordination laws gluing the new activity with sender one. Basically, the activity of the encryption agent is triggered by the presence of tuples representing mail to encrypt mail_to_encrypt (Mail) and produces tuples with mail encrypted mail_encrypted( EncryptedMail). In particular, the encryption agent can get mail_to_encrypt tuples by means of in operations, and provide the mail_encrypted tuples by means of out operations. In order to provide this new feature, we extend the behaviour of the tuple centre with the reactions depicted in Table 2 (top). For hypothesis, the presence of the tuple encryption (true) indicates that the encryption feature is active. According to the extended behaviour, when an outgoing non-encrypted email is inserted in the tuple centre and the encryption service is on, the tuple with the information in clear is removed and the a mail_to_encrypt tuple inserted (reaction 1). Then, a free encryption agent would retrieve the tuple by means of an in or an inp primitive, do its job and then insert the resulting mail_encrypted tuple in the tuple centre. The tuple centre would react to this action, by removing the mail_encrypted tuple and inserting a new outgoing tuple, containing the encrypted email (reaction 2). As in the previous case, the outgoing tuple would be then collected and sent away by the sender agent.

An analogous approach can be adopted for the decryption feature. As for the encryption, we extend the behaviour with suitable coordination laws (see Table 2 , bottom) and introduce a new agent able to decrypt the information (or we can assign the decryption task to the same encryption agent). Analogously to the encryption agent, the activity of the decryption agent is triggered by the pres- 


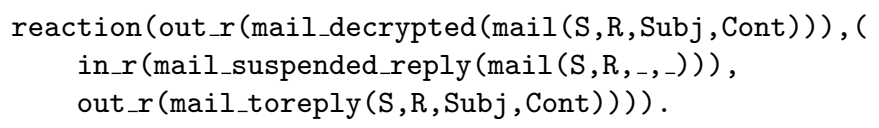

Table 3. ReSpecT code defining the auto-reply feature (top) and code required to avoid feature interaction with the encryption/decryption feature (bottom).

ence of tuples representing mails to decrypt mail_to_decrypt (EncryptedMai $\iota$ ) and produces tuples mail_decrypted(Mai $\iota)$ with mails decrypted. When an incoming tuple is inserted with an encrypted mail (in the form mail $(S, R$, encrypted $(S u b j)$, encrypted $(C))$ ), the tuple is removed and a tuple mail_to_decrypt inserted (reaction 3 ). Then the decryption agent comes into play, consuming the mail_to_decrypt, doing its decryption job and then inserting the new decrypted tuple, mail_decrypted. The tuple centre reacts by removing the mail_decrypted tuple and inserting a new incoming tuple, containing the decrypted email (reaction 4). The incoming tuple is then collected by the user agent and properly visualised.

What is important to note here is that the dynamic/runtime integration of the new encryption and decryption feature has not caused the intervention on existing running agents, but only the extension of the behaviour of coordination artifact with new coordination laws and the introduction of new specialised agents.

\subsection{Handling Feature Interaction: Encryption/Decryption plus Auto-Reply}

Now suppose that we want to add the auto-reply feature, enabling automatic answers to all incoming emails. As remarked in literature, this feature and the encryption/decryption feature interfere [19]. For instance the encryption and auto-responder interact as follows: the auto-responder answers emails automatically by quoting the subject field of the incoming email; if an encrypted email is 
decrypted first and then processed by the auto-responder, an email with the subject of the email will be returned. This however leaks of the originally encrypted subject if the outgoing email is sent in plain.

As for the encryption and decryption feature, we can integrate the new feature by extending the behaviour of the tuple centre with proper coordination laws (see Table 3, top) and by introducing a new agent able to compose the answer mail from the incoming one. The activity of this auto-responder agent is triggered by the presence of tuples mail_toreply(Mai $\iota)$ representing mails to be answered and produces tuples mail_reply (ReplyMai $\iota$ ) with the replies. When an incoming tuple is inserted and the auto-reply feature is active (a tuple auto_reply(true) is present in the tuple set), the tuple mail_toreply is inserted in the tuple centre (reaction 1). Then the auto-responder agent comes into play, consuming the mail_toreply, doing its job - in this simple case creating a reply mail by quoting the subject of the original one - and then inserting the new reply tuple, mail_reply. The tuple centre reacts by removing the mail_reply tuple and inserting a new outgoing tuple, with the reply mail to be sent (reaction 2). The outgoing tuple would be finally collected and sent away by the sender agent.

However, It is easy to verify that this solution suffers the feature interaction problem. The problems arise with incoming encrypted emails, which require to receive an encrypted auto-reply. In this case, the auto-responder agent would be asked to create a reply from an encrypted email, and then to be able to decrypt it first. It is worth noting that there are no problems or interference with the outgoing emails: when the auto-reply agent inserts a non-encrypted outgoing reply, it is collected and encrypted by the encryption agent, and then sent away by the sender agent. In order to decrypt the mail the auto-responder agent could use the available decryption service, by inserting a suitable mail_to_decrypt and then collecting the tuple mail_decrypted as a result. This could be a solution, however in this way we would create a coupling between the auto-responder agent with the decryption service, that is the auto-reply agent must be aware of the existence of encrypted emails and of the related service for their decryption. The problem can be solved by focusing completely on the coordination part, that is adapting the glue among the activities. Our goal is to trigger the decryption activity before the encrypted mail would be collected by the autoresponder agent. To do this we can either modify the reactions added for the auto-reply feature, in order to have a different coordination behaviour if the encryption/decryption feature is active; however this would mean changing the glue related to a feature because of the presence of another feature. Or, we can avoid any change to the existent glue and solving the problem by adding the reactions useful to manage the dependencies between the coordination laws related to the two features, by constraining the execution order of the decryption and auto-reply activity. The reactions are shown in Table 3 (bottom). When the auto-reply service is triggered by inserting a mail_toreply tuple with an encrypted mail, the tuple centre reacts and the tuple is removed - to avoid the immediate service triggering - and a new mail_suspended_reply tuple inserted 
to keep track of the suspension (reaction 3). Then, when the decryption service finishes and the incoming tuple is inserted with the decrypted email, related to a suspended reply, the mail_suspended_reply is removed and the auto-reply service is triggered again by inserting the proper mail_toreply tuple.

So, what is important to remark here is that the feature interaction could be managed without any intervention on the agents responsible on the individual activities, but only by adapting or, possibly, extending the glue that binds the activities together. Of course, the solution (interaction protocols and reactions) provided in this example are useful only in principle, for reasoning about the problem: real-world systems would require more solid and well-engineered tuple formats, protocols and reactions.

\section{Conclusions}

In this paper we investigated the relationships between feature based system and agent oriented software engineering, in particular we sketched out a possible way to model and design feature-based systems in terms of agents and coordination artifacts. Some benefits of the approach have been remarked, in particular the support provided by agents and artifacts to feature runtime integration and evolution, and for the management of features in the context of concurrent and distributed systems. The bridge between the two worlds has triggered some interesting challenges coming from the feature context - such as the feature interaction problem: we found these issues very interesting and challenging also using agents for the system engineering, and should be subject of future investigations.

\section{Acknoledgment}

I would like to thank my professor Andrea Omicini, whose support and ideas have been fundamental first for participating to the Dagstuhl event and then for conceiving this article.

\section{References}

1. M. Calder, M. Kolberg, E. H. Magill, and S. Reiff-Marganiec. Feature Interaction: a critical review and considered forecast. Computer Networks, 41:115-142, 2003.

2. P. Ciancarini, A. Omicini, and F. Zambonelli. Multiagent system engineering: the coordination viewpoint. In N. R. Jennings and Y. Lespérance, editors, Intelligent Agents VI - Agent Theories, Architectures, and Languages, volume 1767 of LNAI, pages 250-259. Springer-Verlag, Feb. 2000.

3. E. Denti, A. Natali, and A. Omicini. On the expressive power of a language for programming coordination media. In Proc. of the 1998 ACM Symposium on Applied Computing (SAC'98), pages 169-177. ACM, 1998. Track on Coordination Models, Languages and Applications. 
4. L. Gasser. MAS infrastructure definitions, needs, prospects. In T. Wagner and O. Rana, editors, Infrastructure for Agents, Multi-Agent Systems, and Scalable Multi-Agent Systems, volume 1887 of LNCS, pages 1-11. Springer-Verlag, Jan. 2001.

5. D. Gelernter and N. Carriero. Coordination languages and their significance. Communications of the ACM, 35(2):97-107, Feb. 1992.

6. R. J. Hall. Feature Interactions in Electronic Mail. In IEEE Workshop on Feature Interaction. IOS-press, 2000.

7. N. R. Jennings and S. Bussmann. Agent-based control systems: Why are they suited to engineering complex systems? IEEE Control Systems Magazine, 23(3):6173, June 2003.

8. N. R. Jennings and M. Wooldridge. Agent-oriented software engineering. In F. J. Garijo and M. Boman, editors, Multi-Agent Systems Engineering, volume 1647 of LNAI. Springer-Verlag, 1999. 9th European Workshop on Modelling Autonomous Agents in a Multi-Agent World (MAAMAW'99), Valencia (E), 30 June - 2 July 1999, Proceedings.

9. D. Keck and P. Kuehn. The feature and service interaction problem in telecommunications systems: a survey. IEEE Transaction on Software Engineering, 24(10):779-796, Oct. 1198

10. A. Omicini. SODA: Societies and infrastructures in the analysis and design of agentbased systems. In P. Ciancarini and M. J. Wooldridge, editors, Agent-Oriented Software Engineering, volume 1957 of LNCS, pages 185-193. Springer-Verlag, 2001.

11. A. Omicini and E. Denti. Formal ReSpecT. In Proceedings of the 2000 Joint Conference on Declarative Programming (AGP'00), La Habana (Cuba), 4-7 Dec. 2000 .

12. A. Omicini and E. Denti. From tuple spaces to tuple centres. Science of Computer Programming, 41(3):277-294, Nov. 2001.

13. A. Omicini and S. Ossowski. Objective versus subjective coordination in the engineering of agent systems. In M. Klusch, S. Bergamaschi, P. Edwards, and P. Petta, editors, Intelligent Information Agents: An AgentLink Perspective, volume 2586 of LNAI: State-of-the-Art Survey, pages 179-202. Springer-Verlag, Mar. 2003.

14. A. Omicini and A. Ricci. Reasoning about organisation: Shaping the infrastructure. AI*IA Notizie, XVI(2):7-16, June 2003.

15. A. Omicini, A. Ricci, and M. Viroli. Formal specification and enactment of security policies through Agent Coordination Contexts. In R. Focardi and G. Zavattaro, editors, Security Issues in Coordination Models, Languages and Systems, volume 85(3) of Electronic Notes in Theoretical Computer Science. Elsevier Science B. V., Aug. 2003. 1st International Workshop (SecCo 2003), Eindhoven, The Netherlands, 28-29 June 2003. Proceedings.

16. A. Omicini and F. Zambonelli. Coordination for Internet application development. Autonomous Agents and Multi-Agent Systems, 2(3):251-269, Sept. 1999. Special Issue: Coordination Mechanisms for Web Agents.

17. G. A. Papadopoulos and F. Arbab. Coordination models and languages. Advances in Computers, 46(The Engineering of Large Systems):329-400, August 1998.

18. M. Plath and M. Ryan. Plug-and-play features. In K. Kimbler and L. G. Bouma, editors, Feature Interactions in Telecommunications and Software Systems, pages 150-164. IOS-press, 1998.

19. C. Prehofer. Plug-and-play composition of features and feature interactions with statechard diagrams. In IEEE Workshop on Feature Interaction. IOS-press, 2003. 
20. A. Ricci, A. Omicini, and E. Denti. Activity Theory as a framework for MAS coordination. In P. Petta, R. Tolksdorf, and F. Zambonelli, editors, Engineering Societies in the Agents World III, volume 2577 of LNCS, pages 96-110. SpringerVerlag, Apr. 2003. 3rd International Workshop (ESAW 2002), Madrid, Spain, 16-17 Sept. 2002. Revised Papers.

21. M. Viroli and A. Omicini. Coordination as a service: Ontological and formal foundation. In A. Brogi and J.-M. Jacquet, editors, FOCLASA 2002 - Foundations of Coordination Languages and Software Architecture, volume 68(3) of Electronic Notes in Theoretical Computer Science. Elsevier Science B. V., Mar. 2003. CONCUR 2002 Satellite Workshop, 19 Aug. 2002, Brno, Czech Republic, Proceedings.

22. M. Wooldridge and P. Ciancarini. Agent-oriented software engineering: The state of the art. In Handbook of Software Engineering and Knowledge Engineering. World Scientific Publishing, 2001.

23. M. J. Wooldridge and N. R. Jennings. Intelligent agents: Theory and practice. The Knowledge Engineering Review, 10(2):115-152, 1995.

24. F. Zambonelli, N. R. Jennings, A. Omicini, and M. Wooldridge. Agent-oriented software engineering for internet applications. In A. Omicini, F. Zambonelli, M. Klusch, and R. Tolksdorf, editors, Coordination of Internet Agents: Models, Technologies, and Applications, chapter 13, pages 369-398. Springer-Verlag, Mar. 2001. 